\title{
Has the liver and other visceral organs migrated to its normal position in children with giant omphalocele? A follow-up study with ultrasonography
}

\author{
Floortje Clemens van Eijck • Willemijn M. Klein • \\ Carla Boetes • Daniel C. Aronson • Rene M. H. Wijnen
}

Received: 18 August 2009/Accepted: 8 September 2009/Published online: 29 September 2009

(C) The Author(s) 2009. This article is published with open access at Springerlink.com

\begin{abstract}
Summary This study evaluates whether, on the long run, in patients born with a giant omphalocele, the liver and other solid organs reach their normal position, shape, and size. Seventeen former patients with a giant omphalocele, treated between 1970 and 2004, were included. Physical examination was supplemented with ultrasonography for ventral hernia and precise description of the liver, spleen, and kidneys. The findings were compared with 17 controls matched for age, gender, and body mass index. We found an abnormal position of the liver, spleen, left kidney, and right kidney in eight, six, five, and four patients, respectively. An unprotected liver was present in all 17 patients and in 11 controls, the difference being statistically significant $(p=0.04)$. In ten of the 11
\end{abstract}

F. C. van Eijck · D. C. Aronson - R. M. H. Wijnen Department of Surgery, Division of Paediatric Surgery, Radboud University Nijmegen Medical Centre,

Nijmegen, The Netherlands

\section{W. M. Klein}

Department of Radiology,

Radboud University Nijmegen Medical Centre,

Nijmegen, The Netherlands

C. Boetes

Department of Radiology, Academic Hospital Maastricht, Maastricht, The Netherlands

F. C. van Eijck $(\square)$

Division of Paediatric Surgery,

Radboud University Nijmegen Medical Centre,

P. O. Box 9101, 6500 HB Nijmegen, The Netherlands

e-mail: fcvaneijck@gmail.com

Present Address:

F. C. van Eijck

Department of Surgery, Erasmus Medical Centre Rotterdam,

Rotterdam, The Netherlands patients with an incisional hernia, the liver was located underneath the abdominal defect.

Conclusion In all former patients with a giant omphalocele, an abnormal position of the liver and in the majority of them, an incisional hernia was also found. The liver and sometimes also the spleen and the kidneys do not migrate to their normal position. Exact documentation and good information are important for both the patient and their caretakers in order to avoid liver trauma.

Keywords Giant omphalocele · Ultrasonography · Biometry liver/spleen · Position visceral organs $\cdot$ Hernia

\section{Introduction}

An omphalocele (OC) is a congenital abdominal wall defect at the umbilicus, which can in most cases be closed primarily. More difficult is a giant omphalocele (GOC), defined as an abdominal wall defect larger than $4 \mathrm{~cm}$ and the liver partly extruded in the omphalocele.

Neonates with GOC often have a small underdeveloped peritoneal cavity with a high degree of viscera abdominal disproportion that can prohibit save primary closure. Placing the abdominal contents under pressure leads to an abdominal compartment syndrome with reduction of cardiac output, hypotension and hampered renal perfusion, and often leads to postoperative respiratory failure by the elevation of both diaphragms. Therefore, primarily, nonoperative approach is recommended by several authors, leaving the organs up front in an uncorrected position. Secondarily, the defect can be closed by several different techniques with reposition of the liver and bowel into the abdominal cavity $[1,3,4,6-8,11,16]$. There is little information on the position and size of the liver later in life 
in patients with GOC [17]. In case the liver is ventrally (medial) located, there is an increased risk for liver rupture in case of an abdominal trauma. In addition, for acute abdominal operations, and in case of pregnancy, the position of the liver could be important. The aim of this study is to investigate if the liver and other solid organs eventually migrated to their normal position, regaining their normal shape and size. Furthermore, special attention was paid to the presence of the natural protection of the liver by the chest wall and the strength of the abdominal wall in case of an 'unprotected' liver.

\section{Materials and methods}

Between 1970 and 2004, 22 (nine males and 13 females) surviving patients with GOC were treated at the Radboud University Nijmegen Medical Centre. All patient records, operative reports, and office notes were retrospectively reviewed.

Patients were treated either surgically or conservatively. Initial operative treatment was divided into primary or staged closure of the abdominal wall defect. Initial nonoperative treatment consisted of covering the omphalocele after birth with dry sterile dressings until it was fully epithelialized, and liver and bowel were partly migrated into the abdomen by gravity. Later in life, the abdominal defect was either closed, or an incisional hernia was accepted.

The addresses of the patients were traced, and they were subsequently asked in writing to participate in this study. After informed consent was obtained, the patients were seen at the outpatient clinic. Patient's characteristics, medical history, use of medication and physical examination, anthropometry (weight, height, and body mass index (BMI)), and a digital picture of the abdomen (anterior and lateral) were collected. The sonographic measurements of organ size were performed with a real-time parallel ultrasound (Toshiba Aplio XG, using a curved 3.5 MHz and linear $8.0 \mathrm{MHz}$ probe). All patients were investigated in supine position. Determination of liver size was calculated by measuring the craniocaudal liver extension in three standardised section planes: anterior axillary line (AAL), medioclavicular line (MCL), and sternal line (STL) (Fig. 1) [5].

Index liver size (ILS) was calculated by the following formula; $I L S=\left(A A L^{2}+M C L^{2}+S T L^{2}\right) \cdot 0.2618$ [5]. The grade of unprotected liver was defined as the number of centimetre of the liver beneath the chest boundary at section planes AAL, MCL, and STL. The spleen size was measured by the largest section area in the longitudinal and transverse plane (Fig. 1). All measurements were repeated three times, and the mean score was recorded as

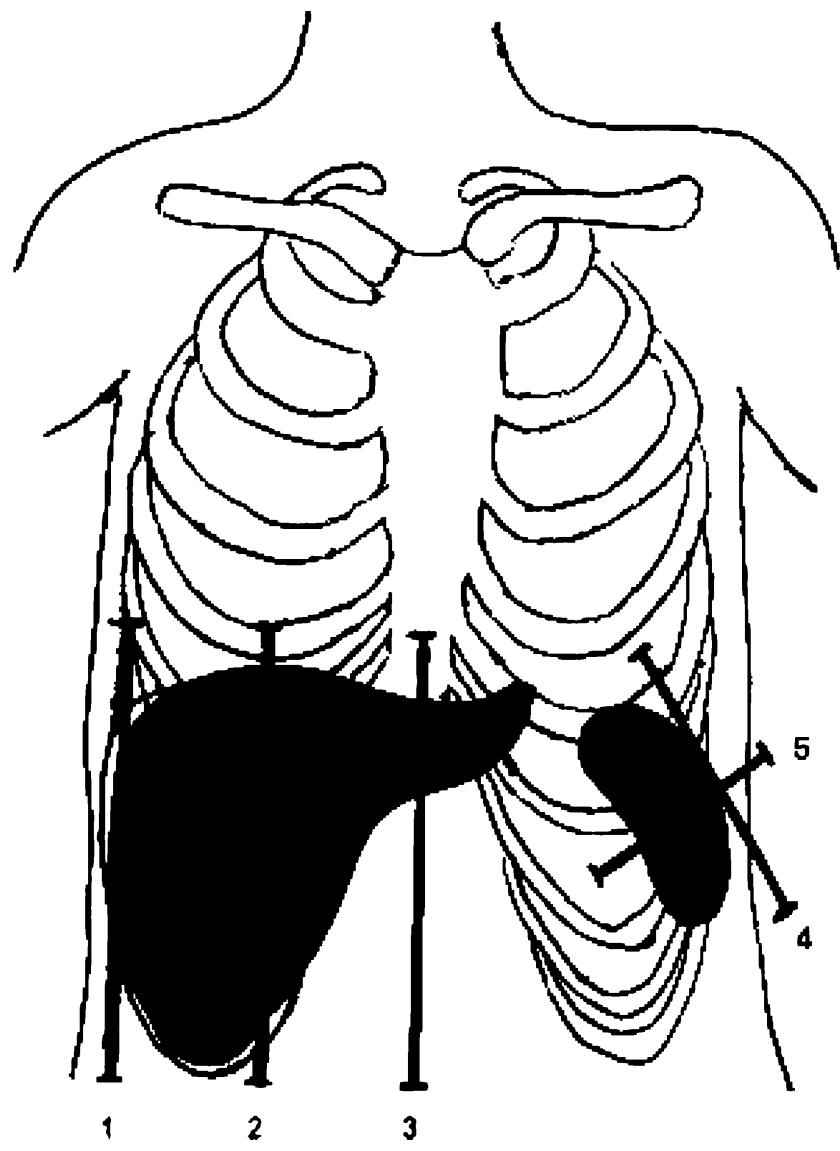

Fig. 1 Section planes of liver and spleen. 1 anterior axillary line (AAL), 2 medioclavicular line (MCL), 3 sternal line (STL), 4 longitudinal section, 5 transverse section

the absolute value. The position of the liver (normal/abnormal position; unprotected liver yes/no; number of centimetre beneath ribcage at section planes AAL, MCL, and STL; thickness of abdominal wall (mm) of unprotected liver), position spleen, kidneys, and the presence of an incisional hernia were recorded.

For peer group reference, the results were compared with a control group (after informed consent), matched for gender, age, and BMI. Significance testing for discrete variables was performed with the Fisher's exact test when appropriate. Student's $t$ test was used for comparing means. Reported $p$ values are two-sided. The $p<0.05$ was considered statistically significant. All analyses were two-sided and conducted using SPSS software (version 16.0; SPSS, Inc., Chicago, Illinois, USA).

\section{Results}

Of the 22 patients, 18 patients in the age from three to 29 years could be traced of whom one did not want to participate in the study. The remaining 17 could be included 
and underwent physical and ultrasonographic examination. Patient's demographics and those of control group showed 12 females and five males in both groups. Median age was 13.3 vs 15 years (range 4-30) and median BMI was 18.4 vs 17.1, respectively (range 13-32). Physical examination did not correlate well with ultrasonography. In only four patients, the liver was palpated during physical examination in contrast to ultrasonography of which all patients had a partly unprotected liver (beneath chest boundaries). Hernia of the abdominal wall was detected during physical examination in nine patients vs 11 hernias that were detected during ultrasound. No correlation was found between patients treated for GOC with primary vs staged closure and presence of hernia ( $7 / 12$ vs $3 / 4)$. In ten out of 11 patients with an incisional hernia, the liver was located underneath the abdominal defect which explained the absence of protrusion of small bowel or fat tissue through the abdominal wall defect during physical examination. The results of the measurements and observations of ultrasound are shown in Table 1. The size of the liver (ILS) was larger in the GOC group $(p=0.01)$. In contrast, the spleen size was equal in both groups. Abnormal position of liver, spleen, left, and right kidney was observed in eight, six, five, and four patients, respectively. Unprotected liver (cm) under the chest boundaries at STL and MCL was significantly more often seen in the GOC group as compared to the control group (Fig. 2). In two patients, the liver was located in the lower abdomen reaching into the pelvis. In these two, one kidney was located cranial from the liver. In one patient the gallbladder was located at the ventral aspect of the liver.

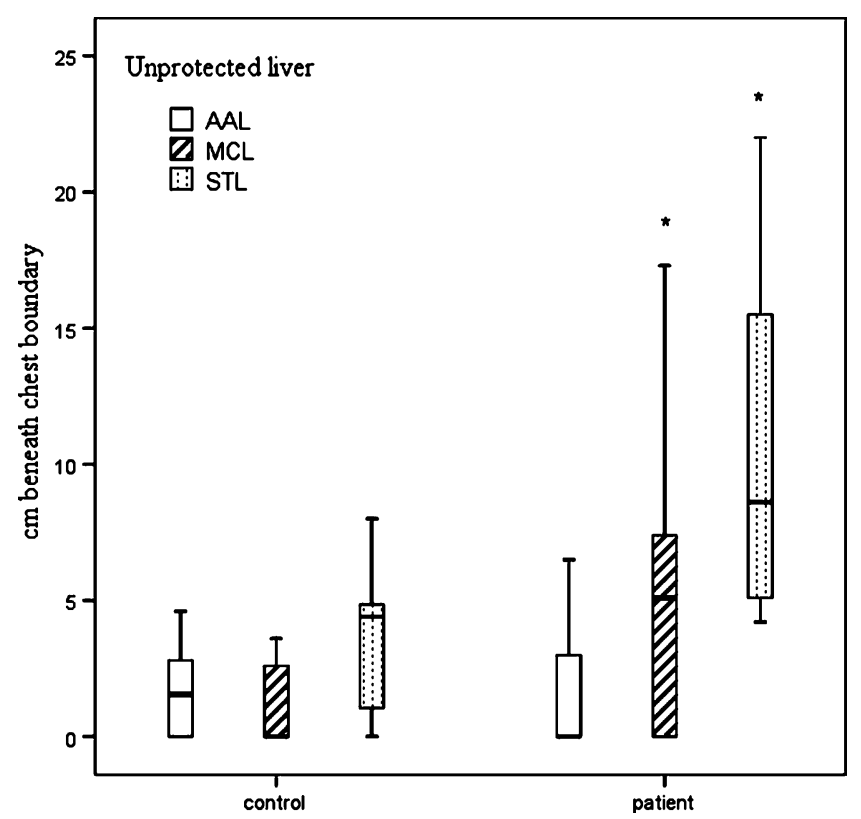

Fig. 2 Unprotected liver beneath chest boundary-patient vs control. Box represents: upper border - first quartile, lower border - third quartile, and black bold line in box represents the median. The asterisk represents the significant difference between patient vs control

\section{Discussion}

The treatment of GOC is still under discussion. In the last decades, several surgical techniques have been described. Primary closure with acceptance of high abdominal pressure gives rise to circulatory problems. Also, in staged closure techniques like silastic silo, skin flap closure can
Table 1 Results measurements ultrasound (median) in 17 patients born with giant omphalocele and 17 controls

\footnotetext{
${ }^{\text {a }}$ Fisher's exact test

${ }^{\mathrm{b}}$ Student's $t$ test
}

\begin{tabular}{lllll}
\hline & & Patient (range) & Control (range) & Sign. \\
\hline Position spleen & Normal & 11 & 17 & $P=0.018^{\mathrm{a}}$ \\
& Abnormal & 6 & 0 & \\
Spleen volume $\left(\mathrm{cm}^{3}\right)$ & & $37.4(6-101)$ & $44(21-93)$ & $P=0.58^{\mathrm{b}}$ \\
Position kidney left & Normal & 12 & 17 & $P=0.044^{\mathrm{a}}$ \\
& Above spleen & 5 & 0 & \\
Position right kidney & Normal & 13 & 17 & $P=0.10^{\mathrm{a}}$ \\
& Above liver & 4 & 0 & \\
Position liver & Normal & 9 & 17 & $P=0.03^{\mathrm{a}}$ \\
& Abnormal & 8 & 0 & $P=0.72^{\mathrm{b}}$ \\
Unprotected liver AAL (cm) & & $0(0-6)$ & $1.6(0-5)$ & $P=0.017^{\mathrm{b}}$ \\
Unprotected liver MCL (cm) & & $5.1(4-22)$ & $0(0-7)$ & $P=0.000^{\mathrm{b}}$ \\
Unprotected liver STL (cm) & & $8.6(0-25)$ & $4.4(0-8)$ & $P=0.012^{\mathrm{b}}$ \\
Index of liver size (ILS) & & $139(59-341)$ & $103(35-180)$ & $P=0.044^{\mathrm{a}}$ \\
Liver totally covered by ribcage & & 0 & 4 & $P=0.31^{\mathrm{b}}$ \\
Thickness abdominal wall at unprotected liver (mm) & $4.5(3-19)$ & $9.5(0-25)$ & $P=0.000^{\mathrm{a}}$ \\
Incisional hernia & Yes & 11 & 0 & 17 \\
Size hernia (cm ${ }^{2}$ ) & No & 6 & - & \\
\hline
\end{tabular}


lead to pulmonary or circulatory problems $[1,2,6,7,9,12$, 16]. Independent of the definitive treatment of closure of the abdominal wall, the position of the liver and of other visceral organs in patients born with GOC differs from the normal position, and it is unclear if the position changes during life. To our knowledge, this is the first study, which demonstrates a persistent abnormal position of liver, kidneys, and spleen in most GOC.

In two patients, the liver was even located in the lower abdomen and pelvis. Incisional hernias were present in 11 patients of which the liver was located in front of the defect in ten, sealing off the defect to such extent that no complaints or symptoms were present. This explains the absence of protrusion of small bowel and/or fat tissue at physical examination.

Zaccara et al. [17] studied the shape and position of liver and spleen in patients with abdominal wall defects (gastroschisis and omphalocele). They found larger index liver size and spleen volume in comparison with normal healthy persons as published by Dittrich et al. [5]. Over the last decennia welfare and most of all, food intake habits have changed anthropometry, which makes it quite difficult to compare these results with data that were obtained 20 years earlier. In our study we used for peer group reference, a control group matched for gender, age, and BMI. There was no difference in spleen volume between the patient group and controls.

Zaccara et al. found a normal position of liver and spleen in patients with abdominal wall defects. In contrast, our study detected an abnormal position of the liver and spleen in eight and six patients, respectively. This difference can be explained by the heterogeneity of the group. In gastroschisis, the liver is not included in the defect. Furthermore, it is unclear if the liver was included in the omphalocele in all patients of their series and not only in GOC.

The high incidence of abdominal wall hernias in our patient group can be explained by high tension still present during primary or staged closure of the abdominal wall. This could plead for initial non-operative treatment after birth and secondary closure if disproportion of the abdominal wall and visceral organs has decreased. Secondary closure using the component separation technique shows promising results with no incisional hernia after a median follow up of 23.5 month [14].

A striking finding was the liver beneath the abdominal wall defect in ten of the 11 patients that theoretically may be quite vulnerable for blunt abdominal trauma. However, literature of traumatic liver rupture in GOC is scarce, and therefore, no evidence is available as to what should be the best treatment option for this specific group.

Even if no herniation was detected, an unprotected liver beneath the ribcage was significantly more present in the patient group as compared to the controls. Studying the ultrasound data, it seems that the volume of the liver is significantly larger and is located more medial and caudal in comparison with the control group. This explains the significant higher grade of unprotected liver.

In literature, there is only one paper describing a perforation of small intestine inside an undetected internal hernia sac after blunt trauma at the age of 40 years [13]. Even in patients with ventral hernia, intestinal perforation after blunt injury seems to be rare $[10,15]$.

In case of acute abdomen or caesarean birth, knowledge of an abnormal position of the liver or of other abdominal organs may be of importance for the choice of the surgical access to the abdomen to prevent damage to the liver and other abdominal organs.

Therefore, good documentation and information of the parents may be of pivotal importance for both patient and surgeon and recommendation of a pre-operative ultrasound should be given. The question remains as to what extent we have to advise exclusion of contact sports and other risk behaviour since information of true increased incidence of blunt trauma in these patients is not available in the literature. However, good information of potential risks to the patients is part of good clinical practise.

Competing interests None of the authors had any conflict of interest.

Open Access This article is distributed under the terms of the Creative Commons Attribution Noncommercial License which permits any noncommercial use, distribution, and reproduction in any medium, provided the original author(s) and source are credited.

\section{References}

1. Allen RG, Wrenn EL Jr (1969) Silon as a sac in the treatment of omphalocele and gastroschisis. J Pediatr Surg 4:3-8

2. Brown MF, Wright L (1998) Delayed external compression reduction of an omphalocele (DECRO): an alternative method of treatment for moderate and large omphaloceles. J Pediatr Surg 33:1113-1115

3. de Lorimier AA, Adzick NS, Harrison MR (1991) Amnion inversion in the treatment of giant omphalocele. J Pediatr Surg 26:804-807

4. De Ugarte DA, Asch MJ, Hedrick MH et al (2004) The use of tissue expanders in the closure of a giant omphalocele. J Pediatr Surg 39:613-615

5. Dittrich M, Milde S, Dinkel E et al (1983) Sonographic biometry of liver and spleen size in childhood. Pediatr Radiol 13:206-211

6. Foglia R, Kane A, Becker D et al (2006) Management of giant omphalocele with rapid creation of abdominal domain. J Pediatr Surg 41:704-709

7. Gross RE (1948) A new method for surgical treatment of large omphalocele. Surgery 24:277-283

8. Nuchtern JG, Baxter R, Hatch EI Jr (1995) Nonoperative initial management versus silon chimney for treatment of giant omphalocele. J Pediatr Surg 30:771-776

9. Pacilli M, Spitz L, Kiely EM et al (2005) Staged repair of giant omphalocele in the neonatal period. J Pediatr Surg 40:785-788 
10. Rab AZ, Fakir SB, PM S (2007) Traumatic ileal perforation in post-traumatic ventral hernia: adding insult to injury. J Coll Physicians Surg Pak 17:756-757

11. Schuster SR (1967) A new method for the staged repair of large omphaloceles. Surg Gynecol Obstet 125:837-850

12. Schwartz MZ, Tyson KR, Milliorn K et al (1983) Staged reduction using a Silastic sac is the treatment of choice for large congenital abdominal wall defects. J Pediatr Surg 18:713-719

13. Seman S, Farber M, Patton J et al (2001) Perforation of small intestine inside an internal omphalocele after blunt trauma: case report and review of the literature. J Trauma 50:343-347
14. van Eijck FC, de Blaauw I, Bleichrodt RP et al (2008) Closure of giant omphaloceles by the abdominal wall component separation technique in infants. J Pediatr Surg 43:246-250

15. Wahab YH, Musa MB (1978) Rupture of herniated small intestine from blunt abdominal injury: a report of 2 cases. Injury 9:225-226

16. Yokomori K, Ohkura M, Kitano Y et al (1992) Advantages and pitfalls of amnion inversion repair for the treatment of large unruptured omphalocele: results of 22 cases. J Pediatr Surg 27:882-884

17. Zaccara A, Iacobelli BD, La SE et al (2003) Sonographic biometry of liver and spleen size long after closure of abdominal wall defects. Eur J Pediatr 162:490-492 